\title{
A SELECTIVE SUMMARY OF PSYCHOPHARMACOLOGY RESEARCH PUBLISHED IN SECOND HALF OF 2017
}

\author{
Manoj Therayil Kumar \\ Director, Institute for Mind and Brain, Inmind, Thrissur. \\ Correspondence: InMind, Minaloor PO, PIN: 680581, Thrissur, Kerala. Email: inmindkerala@gmail.com
}

Psychiatry circles are acutely aware of the lack of breakthroughs in psychopharmacology. Many drugs that showed promise in phase 2 trials have subsequently failed the larger phase 3 trials, and this trend is disturbing. ${ }^{1}$ Phase 3 trials usually involve thousands of patients and in many cases, hundreds of sites. In many of the recently failed trials, the active drug did not separate from placebo due to high placebo response. Financial incentives to recruit more patients by relaxing entry criteria, incompetent use of rating instruments, and defective study design are highlighted as contributing factors. The ongoing stagnation in psychopharmacology would perhaps put pressure on the researchers to have a closer look at these factors. Addressing these could improve research process and thus give new molecules a better chance to reach clinics.

\section{THE 'CANNABIS TREATMENT' FOR PSYCHOSIS}

One-third of patients with schizophrenia show inadequate response to antipsychotics. ${ }^{2}$ Antipsychotics have relatively little impact on negative symptoms and cognitive impairments. ${ }^{3}$ Cannabidiol (CBD) is reported to reduce psychotic symptoms. ${ }^{4,5}$ Mc Guire et al. conducted an exploratory, multicenter, double-blind, randomized, placebo-controlled, parallel-group trial to study the effect of CBD as an adjunctive agent in schizophrenia. ${ }^{6}$ All participants were adults with schizophrenia who were partial responders to a stable dose of one antipsychotic medication for at least four weeks. A score of 60 or more on Positive And Negative Symptom Scale (PANSS) was required to participate in the study. Patients were randomized to receive $1,000 \mathrm{mg} /$ day of CBD (10 $\mathrm{mL}$ of a $100 \mathrm{mg} / \mathrm{mL}$ oral solution) or matching placebo (excipients alone) administered in two divided doses. Eighty-eight patients underwent randomized assignment, and 83 of them completed the trial. Positive psychotic symptoms were significantly reduced from baseline to end of treatment in the CBD group compared with the placebo group. Negative symptoms or overall psychopathology scores were not different between CBD and placebo. Though there were more treatment responders in CBD arm, this fell short of statistical significance. Level of functioning and cognitive performance showed a non-significant improvement in CBD arm. CBD arm had less adverse events, demonstrating its favorable tolerability profile.

This is the first placebo-controlled trial of CBD in schizophrenia. Though the effect on positive symptoms is modest, this is relevant as it has occurred over and above the effect of one antipsychotic agent. There was a trend of overall improvement with CBD. These findings are significant as $\mathrm{CBD}$ is a non-dopamine receptor

Please cite this article as Kumar MT. A selective summary of psychopharmacology research published in second half of 2017. Kerala journal of psychiatry 2017; 30(2):106-14. DOI: 10.30834/KJP.30.2.2018.134 
acting molecule and thus offer the possibility of a new and unique class of antipsychotics.

\section{REFERENCES}

1. Marder SR, Laughren T, Romano SJ. Why are innovative drugs failing in Phase III? Am J Psychiatry 2017; 174(9):829-31.

2. Stone JM, Raffin M, Morrison P, McGuire PK. Review: The biological basis of antipsychotic response in schizophrenia. J Psychopharmacol 2010; 24(7):953-64.

3. Fusar-Poli P, Papanastasiou E, Stahl D, Rocchetti M, Carpenter W, Shergill S, et al. Treatments of negative symptoms in schizophrenia: Meta-Analysis of 168 randomized placebo-controlled trials. Schizophr Bull 2015; 41(4):892-9.

4. Zuardi AW, Crippa JA, Hallak JEC, Pinto JP, Chagas MHN, Rodrigues GGR, et al. Cannabidiol for the treatment of psychosis in Parkinson's disease. J Psychopharmacol 2009; 23(8):979-83.

5. Leweke FM, Piomelli D, Pahlisch F, Muhl D, Gerth CW, Hoyer C, et al. Cannabidiol enhances anandamide signaling and alleviates psychotic symptoms of schizophrenia. Transl Psychiatry 2012; 2: e94.

6. McGuire P, Robson P, Cubala WJ, Vasile D, Morrison PD, Barron R, et al. Cannabidiol (CBD) as an adjunctive therapy in schizophrenia: A multicenter randomized controlled trial. Am J Psychiatry 2017 (Ahead of Print). Available from:

https://doi.org/10.1176/appi.ajp.2017.17030325

\section{PROMISING START FOR 'ANTI SUICIDE' INFUSION}

Ketamine continues to show promising effects in treatment-resistant depression. Rapid relief of mood symptoms following IV ketamine has invited broad interest in its potential use in depression with suicidal risk ${ }^{1}$. Samuel Wilkinson et al. conducted a systematic review to understand the effect of a single dose of ketamine on suicidal ideation. ${ }^{2}$ All placebocontrolled studies with any psychiatric diagnosis were included. Suicidal ideation (both active and passive) was measured by clinician-administered and self-reported instruments at baseline and 1, 2, 3, and seven days post infusion. Ten studies were included in the analysis. Most studies included were of small sample sizes, with a total of 167 patients from 10 studies contributing to this meta-analytic summary.
Results show that suicidal ideas reduced more rapidly in ketamine group. These benefits are apparent from day 1 . These remain significant after controlling for various confounding factors. Ketamine-treated patients were more likely to be free of suicidal ideas. Patients who benefitted in 24 hours were more likely to sustain that effect at day 7. Effect sizes of ketamine on change in suicidal ideation were moderate to large on the self-report outcomes (at day 1, Cohen's d=0.73, 95\% Confidence Interval $(\mathrm{CI})=0.38-1.07$; at day 2, $\mathrm{d}=0.84,95 \% \mathrm{CI}=0.49-1.19$; at day $3, \mathrm{~d}=0.63,95 \%$ $\mathrm{CI}=0.28-0.98$; at day $7, \mathrm{~d}=0.48,95 \% \mathrm{CI}=0.12-$ $0.83)$. The number needed to treat for being free of suicidal ideation was in the range of $3.2-5.0$ for days 1-3 after ketamine infusion, and 9.6 at day 7 .

This meta-analysis shows that ketamine has significant effects on suicidal ideations and that this depends only partly on overall improvement in depressive symptoms. Authors highlight the specific antisuicidal effects of ketamine.

This review provides preliminary evidence that ketamine may have potential as a fast-acting treatment for reducing suicidal thoughts. However, there are several limitations to this conclusion. A single item measure within the depression severity rating scale was used to assess suicidal ideation. This is inadequate for measuring suicide risk. In the real world, a specific, short-acting anti-suicidal agent is more likely to be used in emergency situations where the ambiguity of diagnosis and treatment often exists, and for this, the intended effect needs to be demonstrated across a range of disorders. Specificity on suicidal ideations cannot be claimed as all but one study included in this analysis were among patients with depression.

In spite of accumulating positive evidence, clinicians are reluctant to use ketamine because of the abuse potential and the possible exacerbation of psychosis. Also, so far, no study has looked at ketamine's effect on suicidal behavior (as opposed to suicidal ideas) or the effect on imminent risk of suicide. 


\section{REFERENCES}

1. DiazGranados N, Ibrahim L, Brutsche N, Ameli R, Henter ID, Luckenbaugh DA, et al. Rapid resolution of suicidal ideation after a single infusion of an NMDA antagonist in patients with treatment-resistant major depressive disorder. J Clin Psychiatry 2010; 71(12):160511.

2. Wilkinson ST, Ballard ED, Bloch MH, Mathew SJ, Murrough JW, Feder A, et al. The effect of a single dose of intravenous ketamine on suicidal ideation: A systematic review and individual participant data metaanalysis. Am J Psychiatry 2018;175(2):150-8.

\section{ECT INCREASES BRAIN VOLUME}

Electro Convulsive Therapy (ECT) is viewed by many as a less desirable treatment, despite being the most effective treatment for resistant depression. ${ }^{1}$ Many recent studies suggest that ECT may aid neural plasticity. ${ }^{2}$

Tokamiya et al. systematically reviewed the MRI studies investigating structural changes due to ECT in patients with depression (major depression or bipolar affective disorder) and quantitatively analysed, through a meta-analytic approach, whether ECT induced hippocampal and other brain region structural changes. ${ }^{3}$ They carried out a comprehensive search to locate all studies that used longitudinal designs with at least two scans (before and after ECT). The analysis included 18 studies that met all the inclusion criteria. Changes in left and right hippocampal volume following ECT were designated as primary outcomes, and the secondary outcomes were changes in left and right amygdala volume. There were significant hippocampal and amygdalar volume increases on both sides. Subgroup analysis showed that left hippocampal volume increased in both medicated and unmedicated groups following ECT but was more pronounced in the unmedicated group. The volume increase was more prominent in younger patients. Increased neurogenesis and gliogenesis in the dentate gyrus might explain these hippocampal volume changes. Such changes have been reported with antidepressants, and this would explain the observation that unmedicated patients had greater hippocampal volume change. The clinical relevance of hippocampal volume change remains unclear as there was a negative correlation between clinical improvement and left hippocampal volume increase. This is inconsistent with the literature that, in general, supports hippocampal volume increase with clinical improvement. ${ }^{4}$ This is an interesting observation and would require further studies to ascertain its clinical relevance.

\section{REFERENCES}

1. Lauber C, Nordt C, Falcato L, Rössler W. Can a seizure help? The public's attitude toward electroconvulsive therapy. Psychiatry Res 2005;134(2):205-9.

2. Joshi SH, Espinoza RT, Pirnia T, Shi J, Wang Y, Ayers $\mathrm{B}$, et al. Structural plasticity of the hippocampus and amygdala induced by Electroconvulsive Therapy in major depression. Biol Psychiatry 2016; 79(4):282-92.

3. Takamiya A, Chung JK, Liang K, Graff-Guerrero A, Mimura M, Kishimoto T. Effect of electroconvulsive therapy on hippocampal and amygdala volumes: systematic review and meta-analysis. $\mathrm{Br} \mathrm{J}$ Psychiatry 2018; 212(1):19-26.

4. Arnone D, McKie S, Elliott R, Juhasz G, Thomas EJ, Downey D, et al. State-dependent changes in hippocampal grey matter in depression. Mol Psychiatry 2013; 18(12):1265-72.

\section{WHICH MEDICATION TO GO FOR IN DEMENTIA?}

Donepezil, galantamine, and rivastigmine are the widely used Choline Esterase Inhibitors (CEIs) for Alzheimer's Dementia (AD). Most guidelines suggest CEIs as the first-line pharmacological treatment for mild to moderate AD, jointly with nonpharmacological treatments. ${ }^{1}$ Risk-benefit analysis takes center-stage in prescribing these medications, as benefits may not be substantial and financial burden can be enormous. All-cause discontinuation rate is a pragmatic measure that captures both risks and benefits. Blanco-silevente et al. conducted a meta-analysis of double-blind, randomized placebo-controlled trials of CEIs in 
AD. ${ }^{2}$ The primary outcome measure was all-cause discontinuation. Forty-three studies that provided 60 drug-placebo comparisons were included in this analysis. Most studies excluded dementias other than AD. A total of 16,106 patients with AD were enrolled, and 9,555 received CEIs and 6,551 placebos. No study was concluded to be at high risk of bias.

All-cause discontinuation was higher with CEIs than with placebo (OR $=1.66,95 \%$ CI 1.30-2.03). CEIs were more effective than placebo in reducing cognitive symptoms (SMD $=0.38,95 \%$ CI 0.28 0.47). Drugs did not improve neuropsychiatric symptoms (SMD $=0.03,95 \%$ CI -0.04-0.09). CEIs slightly improved the global symptoms (SMD = $0.28,95 \%$ CI $0.22-0.34$ ). A very small effect was found on functional capacity (SMD $=0.16,95 \% \mathrm{CI}$ 0.11-0.20). Mortality was slightly lower with CEIs than with placebo (OR $=0.65,95 \%$ CI $0.47-0.83)$. CEIs showed modest benefit on cognitive function and global symptomatology in patients with mildmoderate AD.

Analysis of individual medication data suggests that donepezil can be slightly more effective on the global symptomatology of AD than galantamine or rivastigmine. Donepezil and galantamine cause less all-cause discontinuation than rivastigmine. Authors suggest donepezil as the CEI of choice although donepezil is slightly worse for neuropsychiatric symptoms. CEIs show a poor riskbenefit relationship, as indicated by small symptom improvement, and higher all-cause discontinuation than placebo.

\section{REFERENCES}

1. National Institute for Health and Clinical Excellence (2016). Donepezil, galantamine, rivastigmine, and memantine for the treatment of Alzheimer's disease. Available from https://www.nice.org.uk/guidance/TA217.

2. Blanco-Silvente L, Castells X, Saez M, Barceló MA, Garre-Olmo J, Vilalta-Franch J, et al. Discontinuation, efficacy, and safety of cholinesterase inhibitors for Alzheimer's Disease: A meta-analysis and meta- regression of 43 randomized clinical trials enrolling 16 106 patients. Int J Neuropsychopharmacol 2017; 20(7):519-28.

\section{ANTI- INFLAMMATORY AGENTS FOR DEPRESSION}

The macrophage theory of depression was first described in 1991.1 This was based on the observation that cytokines produced by macrophages when given to healthy volunteers induced symptoms of depression. The role of cytokines in depression has been extensively studied in the last two decades. Depression is associated with elevated levels of inflammatory markers. ${ }^{2}$ There is clear evidence of neuroinflammation during acute depressive disorders. ${ }^{3}$ It is suggested that such inflammation reduces the serotonin production. It may also increase production of tryptophan catabolites and one such product, quinolinic acid, an endogenous $N$-methyl-Daspartate (NMDA) receptor agonist, is postulated to have a neurotoxic effect via glutamate release. A comprehensive review of the field with particular focus on anti-inflammatory medications is provided by Paula K Feltes et al. ${ }^{4}$ Clinicians would be keen to know whether any anti-inflammatory agent brings meaningful benefit to those with mood disorders. Husain et al. carried out a meta-analysis of all controlled trials (randomized and crossover) that studied the efficacy of anti-inflammatory drugs in improving both depressive and manic symptoms in patients with MDD or bipolar disorder. ${ }^{5}$ The minimum length of therapy for inclusion was one day. Anti-inflammatory treatments were defined as non-steroidal anti-inflammatory drugs (NSAIDs), cyclooxygenase (COX)-2 inhibitors, proinflammatory cytokine inhibitors, $\mathrm{N}$-acetyl cysteine (NAC) and minocycline hydrochloride.

The primary outcome measure was the effect of anti-inflammatory drugs in the treatment of acute mood symptoms. Fourteen studies were included in the meta-analysis. The most studied medication was celecoxib with 11 studies. Three studies investigated 
$\mathrm{N}$-acetyl cysteine. Infliximab, aspirin, and minocycline were examined in one study each.

Six trials ( $n=214$ patients) showed lower posttreatment depressive symptom scores following treatment with anti-inflammatory agents when compared to treatment as usual or placebo with an overall effect size of -0.71 ( $95 \%$ CI -1.24 to -0.17 ). Five of the 12 available studies reported a mean change in depressive symptoms, without a statistically significant antidepressant effect. Antiinflammatory treatment reduced manic symptom scores with an overall effect size of -0.72 (95\% CI -1.31 to -0.13$)$.

Authors also carried out a qualitative review of studies that were not included in the meta-analysis. The emerging picture is that celecoxib may have beneficial antidepressant effects. TNF alpha antagonist inflimaxib did not show a significant antidepressant effect in general, but it is possible that it might be useful in those with high baseline inflammatory markers. Benefits of NAC is unclear from the current literature. The limited available evidence (from one RCT) shows minocycline to have some antidepressant effects.

This review shows that anti-inflammatory treatments may have a beneficial effect on both depressive and manic symptoms. Clinical benefit in bipolar depression is unclear. Studies have used different scales and measures of symptom change making pooling of effects less desirable. Large-scale trials are essential to generate evidence that could guide clinical practice.

\section{REFERENCES}

1. Smith RS. The macrophage theory of depression. Med Hypotheses 1991; 35(4):298-306.

2. Dowlati Y, Herrmann N, Swardfager W, Liu H, Sham L, Reim EK, et al. A meta-analysis of cytokines in major depression. Biol Psychiatry 2010; 67(5):446-57.

3. Setiawan E, Wilson AA, Mizrahi R, Rusjan PM, Miler L, Rajkowska G, et al. Role of translocator protein density, a marker of neuroinflammation, in the brain during major depressive episodes. JAMA Psychiatry 2015; 72(3):268-75.

4. Kopschina Feltes P, Doorduin J, Klein HC, JuárezOrozco LE, Dierckx RA, Moriguchi-Jeckel CM, et al. Anti-inflammatory treatment for major depressive disorder: implications for patients with an elevated immune profile and non-responders to standard antidepressant therapy. J Psychopharmacol 2017; 31(9):1149-65.

5. Husain MI, Strawbridge R, Stokes PR, Young AH. Antiinflammatory treatments for mood disorders: Systematic review and meta-analysis. J Psychopharmacol 2017; 31(9):1137-48.

\section{METFORMIN FOR HYPERPROLACTINAEMIA}

Hyperprolactinemia is a common adverse effect of prolonged antipsychotic use. ${ }^{1}$ It is associated with increased risk of breast cancer and bone fractures (De Hert et al., 2016). ${ }^{2}$ Reducing antipsychotic dose, adding aripiprazole or dopamine agonists, and adding metformin are some of the strategies suggested to manage this unwanted effect. ${ }^{3,4}$ Metformin is the most studied medication intervention for antipsychotic-induced hyperprolactinemia.

Wei- Zheng et al. systematically assessed the efficacy and safety of adjunctive metformin for antipsychotic-related hyperprolactinemia in adults with schizophrenia. ${ }^{5}$ The primary outcome measure was the efficacy of metformin in reducing serum prolactin level and improving prolactin-related symptoms at the endpoint. The comprehensive search identified four RCTs with a total of 509 patients. All RCTs were conducted in China. Three RCTs were classified as of high quality.

Serum prolactin level was significantly less in the metformin group at the endpoint. All-cause discontinuation was similar between the two groups. One study showed that menstruation problems resolved in two-thirds of patients on metformin compared with $5 \%$ in control group. The mean reduction of serum prolactin level is around 
$150 \mathrm{~m} \mathrm{IU} / \mathrm{L}$, which is modest and may not lead to significant improvement in reducing the risk of breast cancer and bone fractures.

This is the first meta-analysis of metformin for antipsychotic-related hyperprolactinemia in schizophrenia patients. Metformin has been already shown to be effective in reducing weight and other risk factors for cardiovascular diseases in clinically stable overweight patients with psychotic disorders. ${ }^{6}$ A comprehensive review of existing assessment and management guidelines is provided by Grigg et al .?

\section{REFERENCES}

1. Montgomery J, Winterbottom E, Jessani M, Kohegyi E, Fulmer J, Seamonds B, et al. Prevalence of hyperprolactinemia in schizophrenia: association with typical and atypical antipsychotic treatment. J Clin Psychiatry 2004; 65(11):1491-8.

2. De Hert M, Peuskens J, Sabbe T, Mitchell AJ, Stubbs B, Neven $\mathrm{P}$, et al. Relationship between prolactin, breast cancer risk, and antipsychotics in patients with schizophrenia: a critical review. Acta Psychiatr Scand 2016; 133(1):5-22.

3. Meng M, Li W, Zhang S, Wang H, Sheng J, Wang J, et al. Using aripiprazole to reduce antipsychotic-induced hyperprolactinemia: meta-analysis of currently available randomized controlled trials. Shanghai Arch Psychiatry 2015; 27(1):4-17.

4. Lee MS, Song HC, An H, Yang J, Ko YH, Jung IK, et al. Effect of bromocriptine on antipsychotic drug-induced hyperprolactinemia: Eight-week randomized, singleblind, placebo-controlled, multicenter study. Psychiatry Clin Neurosci 2010; 64(1):19-27.

5. Zheng W, Yang X-H, Cai D-B, Ungvari GS, Ng CH, Wang N, et al. Adjunctive metformin for antipsychoticrelated hyperprolactinemia: A meta-analysis of randomized controlled trials. J Psychopharmacol 2017; 31(5):625-31.

6. Jarskog LF, Hamer RM, Catellier DJ, Stewart DD, Lavange L, Ray N, et al. Metformin for weight loss and metabolic control in overweight outpatients with schizophrenia and schizoaffective disorder. Am J Psychiatry 2013; 170(9):1032-40.

7. Grigg J, Worsley R, Thew C, Gurvich C, Thomas N, Kulkarni J. Antipsychotic-induced hyperprolactinemia: synthesis of worldwide guidelines and integrated recommendations for assessment, management, and future research. Psychopharmacology 2017; 234(22):3279-97.
TRICYCLICS AND SSRIS: DO THEY DIFFER IN EFFICACY?

It is generally accepted that there is no difference in efficacy between Tricyclic Antidepressants (TCAs) and Serotonin Specific Reuptake Inhibitors (SSRIs). However, this has not been a settled issue, with one meta-analysis showing SSRIs to be significantly less effective than TCAs. ${ }^{1}$

Baldessarini and Undurranga conducted a most upto-date systematic review of all existing research involving head-to-head, direct comparisons of SSRIs and TCA-like antidepressants reported between 1980 and 2016. ${ }^{2}$ They included doubleblind, head-to-head trials involving at least one TCA versus one SSRI, in adult acute unipolar depression. Antidepressant doses could be fixed or flexible, and total average daily doses were converted to imipramine- equivalents. Main outcome measures were a response (defined as $\geq 50 \%$ reduction in initial depression rating-scale scores) and percent-improvement in measures of change in within-subject depression ratings to the point of last assessment. Eighty-nine reports met the inclusion criteria. They involved a total of 15,435 participants (8,002 with SSRIs, 7,433 with TCAs). In both outcomes, there were very small, nonsignificant, differences in responses between drugtypes $(3.0 \%$ difference in RR and $1.9 \%$ difference in SMD). There were no significant differences among drugs in rates of response or symptom improvement. The all-cause dropout rate was higher with TCAs.

These findings add strong support to the conclusion that differences in efficacy between SSRIs and TCAs among patients with major depressive episodes are small or negligible. SSRIs are first-line antidepressants not just because they are more patient-friendly, but they are as effective as tricyclics. 


\section{REFERENCES}

1. Undurraga J, Baldessarini RJ. Randomized, placebocontrolled trials of antidepressants for acute major depression: thirty-year meta-analytic review. Neuropsychopharmacol Off Publ Am Coll Neuropsychopharmacol 2012; 37(4):851-64.

2. Undurraga J, Baldessarini RJ. Direct comparison of tricyclic and serotonin-reuptake inhibitor antidepressants in randomized head-to-head trials in acute major depression: Systematic review and metaanalysis. J Psychopharmacol 2017; 31(9):1184-9.

\section{PSYCHOTROPICS IN PREGNANCY}

British Association of Psychopharmacology (BAP) published new guidance on the use of psychotropic medications during pregnancy. ${ }^{1}$ BAP comprehensively reviewed the existing literature to produce this most recent guideline.

Pregnancy is not protective against any mental disorder, and the untreated illness is associated with poor maternal and child outcomes. Discontinuing psychotropic medications during pregnancy can also be associated with greater risk of relapse, especially for those who are treated in the psychiatric settings. The guideline also emphasizes that poor mental health in pregnancy is a strong predictor of mental illness postpartum. Evidence also shows that babies born to mothers with depression and treated with antidepressants during pregnancy may have better long-term emotional and behavioral outcomes compared to babies born to mothers with untreated depression.

Management of mental illness during this period is essentially one of collaboration between the prescriber and the patient as evidence is lacking in many areas and the risks are hard to predict. The guidance provides detailed recommendations on how to manage this conversation. Among antidepressants, sertraline has the lowest reported levels of exposure-related adverse effects. All SSRIs may be associated with an increased risk of postpartum hemorrhage, but the clinical significance of this is uncertain as the absolute risk is very low. The risk for cardiac malformations with SSRI, in particular with paroxetine, is not significant when all confounders are taken into account.

There is no convincing evidence to suggest an increased risk of birth defects associated with benzodiazepines or $\mathrm{Z}$ drugs. There is an insufficient number of studies on anxiolytics in pregnancy. Zolpidem may increase the risk of adverse pregnancy outcomes, but the magnitude of this is uncertain.

Among Second-Generation Antipsychotics (SGA), quetiapine, olanzapine, and risperidone are considered to be safe during pregnancy. Among First-Generation Antipsychotics (FGAs), haloperidol has the most safety data. Quetiapine has a low rate of placental passage. There is little evidence for significant risk to maternal and infant outcomes for antipsychotics. An increased risk of malformations following in utero exposure to risperidone cannot be completely ruled out. Switching medications is generally not advisable due to the higher risk of relapse during pregnancy. The guideline suggests using the same antipsychotics that has worked best for the woman, after discussing benefits and risk. Breastfeeding while on clozapine is contraindicated.

There are no known effects of lithium on fertility in females. Existing evidence cannot exclude an effect on cardiovascular anomalies; however, this risk is likely to be small. Valproate is associated with polycystic ovary syndrome and a significant increase in major congenital malformations. Valproate is not to be used in female children, adolescents or women. Carbamazepine can also cause congenital malformations, but a precise estimate of risk is not available. Lamotrigine is not known to cause congenital malformations; however, it passes in high concentration in breast milk and hence, uses while breastfeeding is to be avoided. 
The guideline also provides detailed recommendations on the management of specific disorders in the perinatal period.

\section{REFERENCES}

1. McAllister-Williams RH, Baldwin DS, Cantwell R, Easter A, Gilvarry E, Glover V, et al. British Association for Psychopharmacology consensus guidance on the use of psychotropic medication preconception, in pregnancy and postpartum 2017. J Psychopharmacol 2017; 31(5):519-52.

\section{ANTIPSYCHOTICS — ARE THEY LOSING EFFICACY?}

Has antipsychotic drug efficacy decreased over the decades? If so, why is this happening? Is this because newer antipsychotics are not as effective as older ones?

Stefan Leucht et al. conducted a systematic review and meta-analysis of all placebo-controlled trials in patients with acute exacerbations of schizophrenia or related disorders over the last 60 years. ${ }^{1}$ Clozapine and intramuscular preparations were excluded from this review. All Chinese studies were excluded due to quality concerns. Mean overall change in symptoms on symptom rating scale (PANSS or BPRS) was the primary outcome measure.

This analysis included 167 studies published from 1955 to 2016 with 28,102 participants. The mean duration of illness was 13.4 (Standard Deviation [SD] of 4.7) years; the mean age was 38.7 (SD 5.5) years. Nearly half of studies were sponsored by the manufacturers of medications.

The mean effect size of all studies combined was 0.47 (95\% CI: $0.42-0.51$ ). This translates into a difference of nearly 10 points on PANSS. Patients treated with antipsychotics were twice as likely to respond than those on placebo. The number needed to treat (NNT) for antipsychotics was 6. Half of all patients treated with antipsychotics showed at least a minimal response compared to $30 \%$ on placebo. Nearly a quarter of patients on antipsychotics showed a good response (50\% reduction in scale score) compared to $14 \%$ on placebo, giving an NNT of 8 . The effect size for negative symptoms was smaller when compared to positive symptoms (SMD 0.35 vs 0.45).

Univariate analyses showed that drug-placebo differences decreased over time, with an average rate of 0.08 effect size units per decade. Larger sample sizes were associated with smaller effect size. In multivariate meta-regression, both degrees of placebo response and industry sponsorship contributed to lower effect sizes. Increasing placebo response has contributed to the decreasing effect sizes over time. Sample sizes have increased continually over the years, and the accompanying recruitment pressure and variability among participants have reduced the effect sizes. Authors suggest that smaller studies with better patient selection are the answer to the disturbing observation of decreasing difference between placebo and drug.

\section{REFERENCES}

1. Leucht S, Leucht C, Huhn M, Chaimani A, Mavridis D, Helfer B, et al. Sixty Years of Placebo-Controlled Antipsychotic Drug Trials in Acute Schizophrenia: Systematic Review, Bayesian Meta-Analysis, and MetaRegression of Efficacy Predictors. Am J Psychiatry 2017;174(10):927-42.

\section{PROSPECT OF CARIPRAZINE}

Cariprazine was approved as an antipsychotic agent by FDA in 2015. It is a partial agonist at dopamine D2, D3 and serotonin 5-HT1A receptors and an antagonist at serotonergic 5-HT2B receptors. Its receptor profile is similar to aripiprazole and brexpiprazole. Cariprazine has a preference for D3 receptors, which is considered to be of potential benefit in reducing negative symptoms. Is it living up to that expectations? Jonathan R Scarff reviewed 
the prospects of cariprazine in the treatment of schizophrenia. ${ }^{1}$

Animal studies have shown that cariprazine can increase prosocial behavior. A randomized, placebo-controlled, double-blind trial demonstrated that negative symptoms improved significantly in patients receiving cariprazine compared with patients receiving risperidone. ${ }^{2}$ D2 partial agonism of cariprazine is thought to make it a beneficial agent for those with comorbid substance use disorders. Animal studies have already shown that it can reduce the rewarding effect of cocaine. However, this has not been studied in humans. Another potential advantage of cariprazine is that it has a half-life of 1-3 weeks, which is much longer than that of other antipsychotic agents. This is particularly useful as missed doses may not matter much. The disadvantage of longer half-life is that any adverse effects experienced by patients will persist for a longer duration following dose reduction or discontinuation. A recent metaanalysis found that only amisulpride outperformed placebo in the treatment of predominant negative symptoms. $^{3} \quad$ Another recent meta-analysis investigating the role of cariprazine in acute exacerbation of schizophrenia found a significant positive effect on the PANSS negative as well as positive scores. ${ }^{4}$ There are limited options to treat negative symptoms, and medications that show any benefit in negative symptoms need further attention from researchers and clinicians.

\section{REFERENCES}

1. Scarff JR. The prospects of cariprazine in the treatment of schizophrenia. Ther Adv Psychopharmacol 2017; 7(11):237-9.

2. Németh G, Laszlovszky I, Czobor P, Szalai E, Szatmári $\mathrm{B}$, Harsányi J, et al. Cariprazine versus risperidone monotherapy for treatment of predominant negative symptoms in patients with schizophrenia: a randomized, double-blind, controlled trial. The Lancet 2017; 389(10074):1103-13.

3. Fusar-Poli P, Papanastasiou E, Stahl D, Rocchetti M, Carpenter W, Shergill S, et al. Treatments of negative symptoms in schizophrenia: Meta-Analysis of 168 randomized placebo-controlled trials. Schizophr Bull 2015; 41(4):892-9.

4. Zhao M-J, Qin B, Wang J-B, Zhang Y-P, Zhao J-T, Mao $Y-G$, et al. Efficacy and acceptability of cariprazine in acute exacerbation of schizophrenia: meta-analysis of randomized placebo-controlled trials. J Clin Psychopharmacol 2018; 38(1):55-9.

\footnotetext{
Source of support: None

Conflict of interest: None declared
}

First submitted: $16^{\text {th }}$ February 2018

Published online: $23^{\text {rd }}$ February 2018 